\title{
Perspective
}

\section{Efficacy-oriented compatibility for component-based Chinese medicine}

\author{
Jun-hua ZHANG ${ }^{1}$, Yan ZHU ${ }^{1}$, Xiao-hui FAN², Bo-li ZHANG ${ }^{1,3, *}$ \\ ${ }^{1}$ Tianjin State Key Laboratory of Modern Chinese Medicine, Tianjin University of Traditional Chinese Medicine, Tianjin 300193, China; \\ ${ }^{2}$ Pharmaceutical Informatics Institute, Zhejiang University, Hangzhou 310058, China; ${ }^{3}$ Chinese Academy of Chinese Medical Science, \\ Beijing 100700, China
}

\begin{abstract}
Single-target drugs have not achieved satisfactory therapeutic effects for complex diseases involving multiple factors. Instead, innovations in recent drug research and development have revealed the emergence of compound drugs, such as cocktail therapies and "polypills", as the frontier in new drug development. A traditional Chinese medicine (TCM) prescription that is usually composed of several medicinal herbs can serve a typical representative of compound medicines. Although the traditional compatibility theory of TCM cannot be well expressed using modern scientific language nowadays, the fundamental purpose of TCM compatibility can be understood as promoting efficacy and reducing toxicity. This paper introduces the theory and methods of efficacy-oriented compatibility for developing component-based Chinese medicines.
\end{abstract}

Keywords: efficacy-oriented compatibility; component-based Chinese medicine; traditional Chinese medicine; multi-target drug

Acta Pharmacologica Sinica (2015) 36: 654-658; doi: 10.1038/aps.2015.8; published online 13 Apr 2015

\section{Introduction}

Human disease patterns have changed drastically in recent decades. At present, chronic complex diseases such as coronary heart disease, stroke, diabetes, cancer and chronic obstructive pulmonary disease are common and drain large amounts of healthcare resources. As a consequence, medical purposes and models have been transformed. Recent advances in health sciences have revealed accumulating evidence of the limitations of the current one-drug-one-target lock-and-key model ${ }^{[1]}$. As a result, combination therapies, such as multi-component drugs for multiple targets, are gaining increasing attention and are considered as the next paradigm in drug discovery ${ }^{[1,2]}$. For example, Caduet, a combination of amlodipine and atorvastatin calcium, was approved by the US Food and Drug Administration (FDA) in 2004 for the treatment of hypertension and hypercholesterolemia.

Traditional Chinese medicine (TCM) prescriptions, which are usually composed of several medicinal herbs, are typical representative of compound medicines. After thousands of years of clinical practice, a large number of TCM treatments have been demonstrated to be of significant efficacy and proven safety. In contrast to the simple addition of several

\footnotetext{
* To whom correspondence should be addressed.

E-mail zhangbolipr@163.com

Received 2014-11-12 Accepted 2015-03-03
}

compounds to form a superpill, a TCM prescription follows the principle of compatibility (peiwu) instead of simple stack of herbs. The compatibility principles of TCM prescriptions include considerations of herbal property (hot, cold, warm and cool), herbal taste (acidic, sweet, bitter, pungent and salty), and trend of drug action (meridian entry, up and down, floatation and sinking, and open and close). Although TCM theories are difficult to be expressed in modern scientific language, the fundamental purpose of TCM compatibility is clear and is the same as that of modern medicine: to increase efficacy and reduce toxicity.

\section{Component-based Chinese medicine}

The basic and most important questions closely related to understanding and guiding the clinical practice of TCM are the effective composition and the mechanism of action of a TCM prescription. However, neither question has been well elucidated due to their complexity. Moreover, it remains an insurmountable challenge to clarify the active materials and mechanisms of herbal decoctions or preparations made from Chinese medicinal materials. Furthermore, the quality standards of TCM preparations are not well established, especially regarding the stability and homogeneity among batches. To understand how TCM treats disease and to improve the quality control level, the model and approach for research and development (R\&D) of new TCM drugs should be innovated. 
TCM preparations, which are made from raw herbal slices according to the compatibility theory of TCM, display certain clinical effects. However, their effective materials and their mechanisms of action and safety are not well established; the quality control level is low; and dosages are based on experience and ancient literature. By contrast, natural drugs are mostly made from extractions of single herbs with a fixed ratio of compounds. Research data regarding the effective materials, mechanisms of action, dosages, efficacy and safety of natural drugs are rich and available. Based on solid research foundation, the quality control of natural drugs is well conducted (Table 1). However, by neglecting the compatibility theory of TCM in clinical experience, natural drugs lose the advantage of compound medicines for complex diseases.

A new approach to develop modern Chinese medicine should combine the strong points of both TCM and natural drugs. Under the support of the National Key Basic Research Project on key scientific problems of TCM prescriptions, we previously proposed the concept of component-based Chinese medicine $(\mathrm{CCM})^{[3]}$.

CCM is a type of modern Chinese medicine made from standard components following the compatibility theory and principles of TCM. The standard components are extractions of medicinal herbs or TCM prescriptions with fixed ingredients and ratios. Standard components, not clearly identified compounds, are a group of active materials made using standardized extraction, separation, and purification methods and technologies.

CCMs have the advantages of both TCM and natural drugs (Table 1); they are guided by the theory and principle of compatibility in TCM yet contain relatively stabile effective substances and active mechanisms. In addition, CCMs are characterized by standardized quality control and stability and validated efficacy, safety and dosage. The active mode of CCMs is multi-component for multiple targets/pathways involved in complex disease pathology. Developing modern Chinese medicine by the compatibility of standard components is innovative in that it maintains the core advantages of TCM and integrates the technology of modern drug design. The model of CCM is suitable for developing new drug of small prescription and for the secondary development of Chinese patent medicines.

\section{Efficacy-oriented compatibility}

There are many processes and techniques for developing CCMs. Composing an ideal prescription is the key step, and efficacy-oriented compatibility is a practical approach. The principles and methods for efficacy-oriented compatibility are summarized below.

\section{Principles}

The principles of efficacy-oriented compatibility are the following: highlighting the primary effect, considering secondary effects and reducing adverse effects. On the basis of clinical experience, the preparation of standard components, analysis of composition-activity relationships, and prescription optimization should follow these principles. In addition, efficacyoriented compatibility is ideal for developing small prescriptions composed of no more than ten components.

\section{Methods}

Generally, efficacy-oriented compatibility should integrate traditional experience with modern techniques. The paradigm and related methods of efficacy-oriented compatibility for CCM are described in Figure 1. The target disease and its pathological stage are firstly defined. Then, data mining methods are used to explore the targets and key nodes related to the disease and to analyze potential effective prescriptions and components. Using methods of network pharmacology and high-throughput screening, the candidate prescription is primarily revised and further evaluated by experimental studies. The candidate prescription will then be optimized according to the principles stated above, which is the key link of efficacy-oriented compatibility. Finally, the optimized prescription should be assessed by clinical trials (Figure 1). Some of the available techniques are summarized below.

\section{Data mining}

Along with the rapid development of science and technology, significant progress has been made in the understanding of life at the cellular and molecular levels. Targets associated with certain diseases have been gradually discovered, and the currently identified targets for disease treatment are countable $^{[4]}$. Potential targets associated with disease are mostly reported in the scientific literature and biological databases. For example, the most popular biomedical literature database, MEDLINE/PubMed, currently contains more than 18 million literature abstracts, and more than 60000 new abstracts are added monthly. Similarly, chemical, genomic, proteomic and metabolic data are collected in the MEDLINE database. Developing in pace with the growth of biological databases, the flourishing of bioinformatics, especially data mining

Table 1. Comparison of three different types of herbal medicines.

\begin{tabular}{|c|c|c|c|c|c|c|c|c|}
\hline Category & Constitution & $\begin{array}{c}\text { Theory } \\
\text { of } \\
\text { TCM }\end{array}$ & $\begin{array}{l}\text { Effective } \\
\text { materials }\end{array}$ & $\begin{array}{l}\text { Mechanism } \\
\text { of action }\end{array}$ & $\begin{array}{l}\text { Quality } \\
\text { control }\end{array}$ & Dosage & Efficacy & Safety \\
\hline Traditional Chinese medicine & Raw herbal slices & Yes & Unclear & Unclear & Low & Experience & Yes & Unclear \\
\hline Natural drug & Extraction with fixed compounds & No & Clear & Clear & High & Research & Yes & Clear \\
\hline Component-based Chinese medicine & Standard components & Yes & Clear & Clear & High & Research & Yes & Clear \\
\hline
\end{tabular}




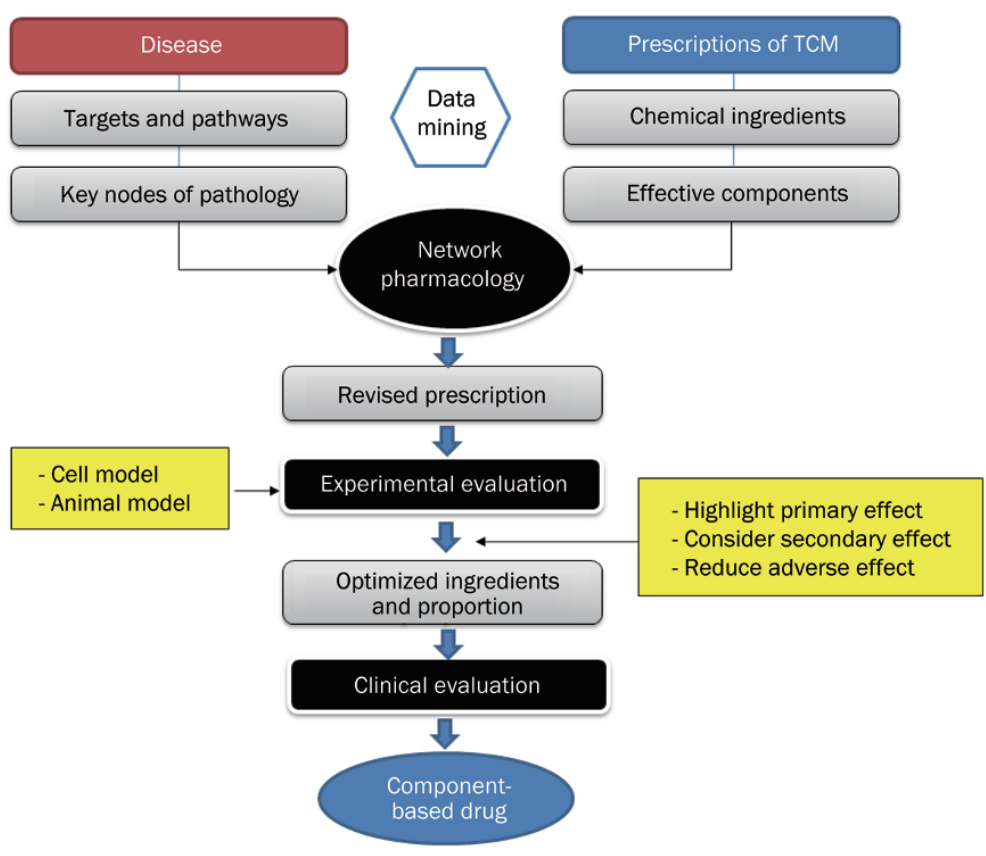

Figure 1. Paradigm of efficacy-oriented compatibility for component-based Chinese medicine (CCM).

approaches, has changed the methods of target discovery ${ }^{[5]}$. Currently, text mining of literature databases and microarray data mining are the two prevailing approaches to target discovery ${ }^{[6]}$. Text mining has been broadly applied to identify disease-associated genes/proteins and to understand their roles in diseases ${ }^{[7,8]}$. The systematic approach is a strategy that selects targets through the study of diseases in whole organisms using information derived from clinical trials and in vivo animal studies. Researchers can identify disease-associated networks and predict key nodes automatically by data mining $^{[9,10]}$. Although data mining is very useful for deriving biological entities and insights from a large number of research articles, it is a preliminary strategy that requires validation by experimental studies.

\section{Database of standard herbal components}

For drug discovery, historical experience is always significant. Indeed, many TCM prescriptions have been used for thousands of years. Thus, CCM should adequately utilize ancient records and clinical experience. With innovations in analytic technology and equipment, it becomes possible and easier to determine the constituents of single herbs or prescriptions. The ingredients of approximately 400 herbs that are commonly used in TCM have been gradually identified. We have established a component materials warehouse and database for herbal components, which is a resource for developing new prescriptions for multiple targets. Specifically, more than 20000 standard herbal components have been extracted from commonly used medicinal herbs and prescriptions.

In cooperation with the TCM research team at Zhejiang University, we developed an in silico approach to predict potential targets of herbal ingredients based on known rela- tionships between FDA-approved drugs and their targets. The performance of predictive models was evaluated by cross-validation and external datasets, which achieved good predictive accuracy. The models were then applied to 10339 TCM ingredients, resulting in 6670 predicted ingredient-target relationships with high confidence. The TCM potential target database (TCM-PTD) is now freely accessible online (http:/ / tcm.zju.edu.cn/ptd).

\section{Network pharmacology}

Network analyses of biological pathways and interactions have revealed that much of the robustness of biological systems is derived from the structure of the network ${ }^{[11,12]}$. Drugs with multiple ingredients aimed at multiple targets may show better effects on the complex equilibrium of whole cellular networks than drugs that act on a single target. Additionally, systematic drug-design strategies should be more efficient than simple combinations of several compounds ${ }^{[13]}$. Integrating network biology and polypharmacology holds the promise of expanding the number of druggable targets. Advances in these areas are creating the foundation of network pharmacology for drug discovery ${ }^{[14]}$. Network analysis does not preclude the identification of individual targets, yet the key challenge facing the development of network pharmacology is identifying a node or combination of nodes in a biological network whose perturbation results in a desired therapeutic outcome $^{[15,16]}$. Biological databases, data mining and databases of herbal components provide the basic conditions for network pharmacology research.

\section{High-throughput screening (HTS)}

Network pharmacological research can develop a candidate 
prescription based on herbal components. The next step is to optimize the components and proportions of the candidate prescription. HTS is a well-established process in discovery for pharmaceutical and biotechnology companies and is increasingly applied to research in academia and medical institutions. HTS has evolved into a mature discipline of modern drug discovery. Tens of thousands to millions of samples are tested in HTS campaigns for their ability to modulate biochemical targets in cell-free assays and/or phenotypic or targeted cell-based assays ${ }^{[17]}$. However, the application of HTS for the identification of biologically active natural products, such as TCM libraries, remains a relatively uncommon activity.

The targets and cellular phenomena amenable to HTS include nuclear receptors, $G$ protein-coupled receptors (GPCRs), ion channels, protein kinases, proteases, signaling pathways, cell death mechanisms and others. For each category of targets commonly subjected to HTS, natural product modulators have been identified ${ }^{[18]}$. A strategy for the production of high-quality fractionated libraries of Chinese herbal formulas for HTS was first introduced by Liu et al ${ }^{[19]}$. A team of US and Chinese co-investigators with expertise in TCM, botany, chemistry and drug discovery has jointly established a prototype library consisting of 202 authenticated medicinal plant and fungal species that collectively represent the therapeutic content of the majority of commonly prescribed TCM herbal prescriptions. Initial screening targets have been applied to preliminary evaluations of 3709 TCM fractions from 82 authenticated TCM species ${ }^{[20]}$.

Developments in techniques for component characterizing, biological evaluation and other screening methods under the perspective of their applicability in natural product have been described in an article by $\mathrm{Zhu}^{[21]}$. In particular, HTS is likely to increase success in modern drug discovery from TCM.

\section{A case}

Myocardial infarction (MI) is a typical polygenic disease. Anti-platelet drugs, beta-blockers, angiotensin-converting enzyme (ACE) inhibitors, and statins have been recommended for clinical practice based on the results of randomized clinical trials and their systematic reviews. However, most of these conventional drugs are based on specific pathways, mainly a single drug acting on a single target. This means that a patient might need to take several drugs concurrently, which leads to new problems, such as low adherence, high cost, and more adverse effects ${ }^{[22]}$. Thus, a new strategy for the management of patients following MI is needed. The concept of "polypill" was developed approximately 12 years ago, with a compound pill including several conventional drugs ${ }^{[23]}$. The Indian Polycap Study showed that a polypill composed of hydrochlorothiazide, atenolol, ramipril, simvastatin and aspirin had the desired effects and was as safe as the individual pills ${ }^{[24]}$. The polypill is a new concept in Western medicine, but it is not new in TCM.

The Qi-Shen-Yi-Qi (QSYQ) pill, a type of polypill for treating $\mathrm{MI}$, is a $\mathrm{CCM}^{[25]}$. QSYQ is composed of extracts from 4 herbs: Radix Astragalus membranaceus (Huangqi), Radix Salvia miltiorrhiza (Danshen), Panax notoginseng (Sanqi) and Lignum Dalbergiae Odoriferae (Jiangxiang). These herbs constitute the QSYQ pill based on efficacy-oriented compatibility principles.

Over the past several years, in vivo and in vitro studies have revealed the integrated effects of QSYQ for MI, including protection of cardiac muscle cells, prevention of cardiac ischemiareperfusion injury via energy modulation, antagonized ventricular remodeling, inhibition of the inflammatory reaction and the progression of atherosclerosis, and stabilization of atherosclerotic plaques through changes in histological constitution $^{[26-32]}$. Recently, a network pharmacology study further revealed the underlying multi-compound, multi-target and multi-pathway mode of action (MOA) of QSYQ ${ }^{[31]}$. This study evidentially confirmed the roles of the QSYQ component herbs in the primary effect of treating MI: Huangqi serves as the sovereign, Danshen as the minister, Sanqi as the assistant and Jiangxiang as the courier ${ }^{[32]}$.

\section{Perspective}

Single-target drugs have showed only limited success for multi-factor diseases, which is why the success rate of new drugs is low despite careful and considerable efforts. The fundamental problem may not be technological, but philosophical: the wrong approach to drug discovery has been followed. In the field of new drug $R \& D$, ideas and methods are undergoing innovation, and compound drug development is becoming the cutting edge in new drug development. In contrast to chemical drug development, which proceeds from the laboratory bench to the clinical bedside, TCM preparations are generated from clinical practice. The development of modern or scientific Chinese drugs should begin with clinical experience and classic prescriptions. The development of modern science and technology provides powerful support for developing new TCM drugs. One key point is that R\&D of new TCM drugs is inseparable from the theory of TCM, especially the theory of prescription compatibility. Efficacy-oriented compatibility is a practical mode for developing CCM, which also needs further development in practice.

\section{Acknowledgements}

This work was supported by the National Key Basic Research Program of China (2012CB518404); the Ministry of Education of China-Program for Innovative Research Team (IRT1276); and the New Century Excellent Talent of Ministry of Education of China (NCET-13-0936).

\section{References}

1 Medina-Franco JL, Giulianotti MA, Welmaker GS, Houghten RA. Shifting from the single to the multitarget paradigm in drug discovery. Drug Discov Today 2013; 18: 495-501.

2 Sams-Dodd F. Target-based drug discovery: is something wrong? Drug Discov Today 2005; 10: 139-47.

3 Zhang Bl, Wang Yy. Theories and Methods Used in the Research of Modern Chinese Medicine by Drug Combination. Chin J Nat Med 2005; 3: 258-61. 
4 Szuromi P, Vinson V, Marshall E. Rethinking drug discovery. Science 2004; 303: 1795.

5 Yang Y, Adelstein SJ, Kassis Al. Target discovery from data mining approaches. Drug Discov Today 2012; 17: S16-23.

6 Sakharkar MK, Sakharkar KR. Targetability of human disease genes. Curr Drug Discov Technol 2007; 4: 48-58.

7 Pospisil P, lyer LK, Adelstein SJ, Kassis Al. A combined approach to data mining of textual and structured data to identify cancer-related targets. BMC Bioinformatics 2006; 7: 354.

8 Ozgür A, Vu T, Erkan G, Radev DR. Identifying gene-disease associations using centrality on a literature mined gene-interaction network. Bioinformatics 2008; 24: i277-85.

9 Krauthammer M, Kaufmann CA, Gilliam TC, Rzhetsky A. Molecular triangulation: bridging linkage and molecular-network information for identifying candidate genes in Alzheimer's disease. Proc Natl Acad Sci U S A 2004; 101: 15148-53.

10 Cheng D, Knox C, Young N, Stothard P, Damaraju S, Wishart DS. PolySearch: a web-based text mining system for extracting relationships between human diseases, genes, mutations, drugs and metabolites. Nucleic Acids Res 2008; 36: W399-405.

11 Albert R, Jeong $\mathrm{H}$, Barabasi AL. Error and attack tolerance of complex networks. Nature 2000; 406: 378-82.

12 Barabási AL, Oltvai ZN. Network biology: understanding the cell's functional organization. Nat Rev Genet 2004; 5: 101-13.

13 Csermely P, Agoston V, Pongor S. The efficiency of multi-target drugs: the network approach might help drug design. Trends Pharmacol Sci 2005; 26: 178-82.

14 Hopkins AL. Network pharmacology: the next paradigm in drug discovery. Nat Chem Biol 2008; 4: 682-90.

15 Mayer LD, Janoff AS. Optimizing combination chemotherapy by controlling drug ratios. Mol Interv 2007; 7: 216-23.

16 Ramaswamy S. Rational design of cancer-drug combinations. N Engl J Med 2007; 357: 299-300.

17 Mayr LM, Fuerst P. The future of high-throughput screening. J Biomol Screen 2008; 13: 443-8.

18 Curtis J. Henrichand John A. Beutler. Matching the power of high throughput screening to the chemical diversity of natural products. Nat Prod Rep 2013; 30: 1284-98.

19 Liu L, Li YF, Cheng YY. A method for the production and characterization of fractionated libraries from Chinese herbal formulas. J Chromatogr B Analyt Technol Biomed Life Sci 2008; 862: 196-204.

20 Eisenberg DM, Harris ES, Littlefield BA, Cao S, Craycroft JA, Scholten $\mathrm{R}$, et al. Developing a library of authenticated Traditional Chinese Medicinal (TCM) plants for systematic biological evaluation-rationale, methods and preliminary results from a Sino-American collaboration. Fitoterapia 2011; 82: 17-33.

21 Zhu Y, Zhang Z, Zhang M, Mais DE, Wang MW, et al. High-throughput screening for bioactive components from traditional Chinese medicine. Comb Chem High Throughput Screen 2010; 13: 837-48.

22 Xing DM, Zhang JH, Li L, Zhu MJ, Shang HC, et al. Intervention effects and safety of cardiovascular Polypill for the relevant risk factors of coronary heart disease: a systematic review. Chin J Evid-based Med 2013; 13: 446-51.

23 Wald NJ, Law MR. A strategy to reduce cardiovascular disease by more than 80\%. BMJ 2003; 326: 1419.

24 Indian Polycap Study (TIPS), Yusuf S, Pais P, Afzal R, Xavier D, Teo $\mathrm{K}$, et al. Effects of a polypill (Polycap) on risk factors in middle-aged individuals without cardiovascular disease (TIPS): a phase II, doubleblind, randomised trial. Lancet 2009; 373: 1341-51.

25 Shang H, Zhang J, Yao C, Liu B, Gao X, Ren M, et al. Qi-shen-yi-qi dripping pills for the secondary prevention of myocardial infarction: a randomised clinical trial. Evid Based Complement Alternat Med 2013; 2013: 738391.

26 Yan FF, Liu Y, Liu YF, Zhao YX. Effect of Qishenyiqi dripping pills on histology of experimental atherosclerotic plaque. J Nanjing Univ Tradit Chin Med 2007; 23: 295-7.

27 Yan FF, Liu Y, Liu YF, Zhao YX. Effects of Qishenyiqi Dripping pills on high sensitivity $C$ reactive protein in experimental atherosclerosis rabbits. Shanghai J Tradit Chin Med 2007; 41: 59-60.

28 Du WX, Zhu MD, Feng LM, Song QG, Wei Y, Ma P, et al. Intervention effect of Qishenyiqi dripping pills on early ventricular remodeling after acute myocardial infarction. Chin J Evid Based Cardiovasc Med 2008; 28: 41-3.

29 Hong C, Wang Y, Lou J, Liu Q, Qu H, Cheng Y. Analysis of myocardial proteomic alteration after Qishenyiqi formula treatment in acute infarcted rat hearts. Zhongguo Zhong Yao Za Zhi 2009; 34: 1018-21.

30 Zhang L, Wang Y, Yu L, Liu L, Qu H, Wang Y, et al. QI-SHEN-YI-QI accelerates angiogenesis after myocardial infarction in rats. Int $J$ Cardiol 2010; 143: 105-9.

31 Li X, Wu L, Liu W, Jin Y, Chen Q, Wang L, et al. A network pharmacology study of Chinese medicine QiShenYiQi to reveal its underlying multi-compound, multi-target, multi-pathway mode of action. PLoS One 2014; 9: e95004.

32 Wu L, Wang Y, Li Z, Zhang B, Cheng Y, Fan X. Identifying roles of "JunChen-Zuo-Shi" component herbs of QiShenYiQi formula in treating acute myocardial ischemia by network pharmacology. Chin Med 2014; 9: 24. 